\title{
Multidegrees of Monomial Rational Maps
}

\author{
by \\ Paolo Aluffi
}

\begin{abstract}
We prove a formula for the multidegrees of a rational map defined by generalized monomials on a projective variety, in terms of integrals over an associated Newton region. This formula leads to an expression of the multidegrees as volumes of related polytopes, in the spirit of the classical Bernstein-Kouchnirenko theorem, extending the scope of these formulas to more general monomial maps. We also determine a condition under which the multidegrees may be computed in terms of the characteristic polynomial of an associated matrix.
\end{abstract}

2010 Mathematics Subject Classification: Primary 14C17; Secondary 14E07.

Keywords: invariants of rational maps, Cremona transformations, Segre classes, volumes of polytopes.

\section{$\S 1$. Introduction}

Let $V \subseteq \mathbb{P}^{r}$ be a projective variety, and $\varphi: V \rightarrow \mathbb{P}^{M}$ a rational map. The multidegrees $\gamma_{\ell}$ of $\varphi, \ell=0, \ldots, \operatorname{dim} V$, are the coefficients of the class of the (closure of the) graph $\Gamma$ of this map in $\mathbb{P}^{r} \times \mathbb{P}^{M}$, to wit

$$
\gamma_{\ell}=h^{\operatorname{dim} V-\ell} \cdot H^{\ell} \cdot \Gamma,
$$

where $h$, resp., $H$ is the pull-back of the hyperplane class in $\mathbb{P}^{r}$, resp., $\mathbb{P}^{M}$. The numbers $\gamma_{\ell}$ are obviously significant: for example, $\gamma_{\operatorname{dim} V}=0$ if and only if the general nonempty fiber of $\varphi$ is positive dimensional; and if the general nonempty fiber consists of $D$ reduced points, then $\gamma_{\operatorname{dim} V}$ is the product of $D$ and $\operatorname{deg}(\overline{\operatorname{im} \varphi})$. When $V=\mathbb{P}^{r}$ and $M=r, \varphi$ is a Cremona transformation if and only if $\gamma_{r}=1$. In general, $\gamma_{0}=\operatorname{deg} V$ and $\gamma_{i}=0$ if and only if $i>\operatorname{dim}(\operatorname{im} \varphi)$; if $V=\mathbb{P}^{r}$ and $i<\operatorname{dim}(\operatorname{im} \varphi), \gamma_{i}$ may be interpreted as the degree of the closure of the image of a general $\mathbb{P}^{i} \subseteq \mathbb{P}^{r}$. We assemble the multidegrees into a polynomial

Communicated by T. Mochizuki. Received January 5, 2015.

P. Aluffi: Mathematics Department, Florida State University, Tallahassee, FL 32306, USA; e-mail: aluffi@math.fsu.edu 


$$
\gamma_{\varphi}(t)=\gamma_{0}+\gamma_{1} t+\gamma_{2} t^{2}+\cdots
$$

of degree $\operatorname{dim}(\operatorname{im} \varphi)$. This polynomial does not depend on the dimension $r$ of the space containing $V$; it does depend on the hyperplane class of the embedding $V \hookrightarrow \mathbb{P}^{r}$. We can in fact define a multidegree class $(\S 2.1)$ on $V$ as the push-forward to $V$ of $\left(\sum_{\ell \geq 0} H^{\ell}\right) \cdot[\Gamma]$; this is independent of any projective embedding of $V$, and our results will in fact deal with this class. In this introduction we will state the results for the multidegree polynomial, to remain closer to the more classical notion. We note that Macaulay2 ([GS]) includes a multidegree command, which is very useful for experimentations involving concrete examples.

We consider rational maps $\varphi$ whose components are monomials $\mu_{0}, \ldots, \mu_{M}$ in sections $s_{j}$ of line bundles $\mathscr{L}_{j}, j=1, \ldots, n$, on $V$, of course subject to the condition that all monomials are sections of the same line bundle $\mathscr{L}$. For example, $\varphi$ could be the restriction to $V$ of a rational map $\mathbb{P}^{r} \rightarrow \mathbb{P}^{M}$ defined by isobaric monomials in a collection of homogeneous polynomials. The hypersurfaces $X_{j}$ defined by $s_{j}$ on $V$ are required to satisfy a weak transversality hypothesis, explained in $\S 2.2$. For simplicity, the reader may assume that $V$ is nonsingular and the $X_{j}$ form a simple normal crossing divisor, but much less is needed: local equations for the divisors $X_{j}$ only need to determine regular sequences, i.e., to meet with regular crossings in the sense of [Har15]; and $V$ is not required to be nonsingular (see §2.2 for further details). We say that $\varphi$ is r.c. monomial if it satisfies this condition.

We now state the result. The monomials $\mu_{i}=s_{1}^{m_{i 1}} \cdots s_{n}^{m_{i n}}$ determine lattice points $\left(m_{i 1}, \ldots, m_{i n}\right)$ in $\mathbb{Z}^{n} \subseteq \mathbb{R}^{n}$ (with coordinates $\left(a_{1}, \ldots, a_{n}\right)$ ). We move this set of points so that one of them is at the origin, by setting $m_{i j}^{\prime}=m_{i j}-m_{M j}$ for $i=0, \ldots, M$. Notice that the lattice points $\underline{m}_{i}^{\prime}=\left(m_{i 1}^{\prime}, \ldots, m_{i n}^{\prime}\right)$ all lie on the subspace $d_{1} a_{1}+\cdots+d_{n} a_{n}=0$, where $d_{j}=h^{\operatorname{dim} V-1} \cdot X_{j}$ is the degree of $X_{j}$ viewed as an algebraic set in $\mathbb{P}^{r}$.

We denote by $N_{\varphi}$ the convex hull of the positive orthants translated at the lattice points $\underline{m}_{i}^{\prime}$; we call $N_{\varphi}$ the Newton outer region of $\varphi$.

Theorem 1.1. Let $\varphi: V \rightarrow \mathbb{P}^{M}$ be a r.c. monomial rational map. Then

$$
\gamma_{\varphi}(t)=\int_{N_{\varphi}} \frac{n ! X_{1} \cdots X_{n} t^{n} h^{\operatorname{dim} V+1} d a_{1} \cdots d a_{n}}{\left(h+\left(a_{1} X_{1}+\cdots+a_{n} X_{n}\right) t\right)^{n+1}} .
$$

Remark 1.2. (i) The integral should be interpreted as follows. Perform the integral with $X_{j}, h$, and $t$ as parameters; the result is a rational function in these parameters. Part of the content of the statement is that after replacing the parameters $X_{j}$ by the classes of the corresponding divisors in $V$, and $h$ by the restriction of the hyperplane class from $\mathbb{P}^{r}$, this rational function gives a polynomial in $t$; the coefficient of $t^{\ell}$ in this polynomial is a homogeneous polynomial of degree $\operatorname{dim} V$ in the 
classes $X_{j}$ and $h$. The statement is that replacing the terms $h^{\operatorname{dim} V-\ell} \cdot X_{j_{1}} \cdots X_{j_{\ell}}$ in this polynomial with the corresponding intersection numbers determines the $\ell$-th multidegree $\gamma_{\ell}$.

(ii) The convex region $N_{\varphi}$ depends on the choice of the pivoting monomial. It is a consequence of the theorem that this choice does not affect the result of evaluating the integral as specified in (i).

Example 1.3. Let $F_{1}, F_{2}, F_{3}$ be general homogeneous polynomials in $x_{0}, x_{1}, x_{2}$ of degrees 1, 2, 3 respectively. Consider the rational map $\varphi: \mathbb{P}^{2} \rightarrow \mathbb{P}^{2}$ given in components by $\left(x_{0}, x_{1}, x_{2}\right) \mapsto\left(F_{2} F_{3}^{2}, F_{1}^{2} F_{3}^{2}, F_{1}^{3} F_{2} F_{3}\right)$. According to Theorem 1.1, the multidegrees of $\varphi$ are the coefficients of $t^{\ell}$ in

$$
\int_{N_{\varphi}} \frac{n ! X_{1} \cdots X_{n} t^{n} h^{\operatorname{dim} V+1} d a_{1} \cdots d a_{n}}{\left(h+\left(a_{1} X_{1}+\cdots+a_{n} X_{n}\right) t\right)^{n+1}}
$$

where $n=3, \operatorname{dim} V=2, X_{1}, X_{2}, X_{3}$ are the curves $F_{1}=0, F_{2}=0, F_{3}=0$, respectively, and $N_{\varphi}$ is the Newton outer region determined by the lattice points $(0,1,2),(2,0,2),(3,1,1)$ translated back to $A=(-3,0,1), B=(-1,-1,1), C=$ $(0,0,0)$. That is, $N_{\varphi}$ is the region in $\mathbb{R}^{3}$ extending from the triangle $A B C$ towards the three positive coordinate directions.

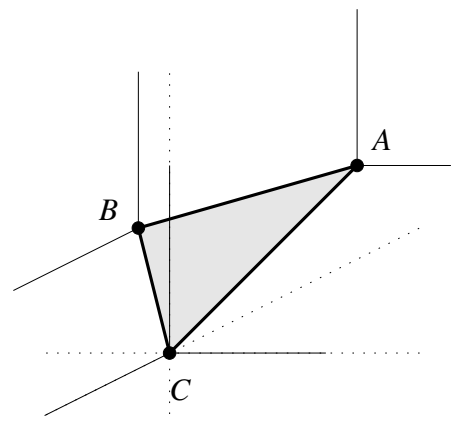

The reader will easily verify that

$$
\begin{aligned}
\int_{N_{\varphi}} & \frac{6 X_{1} X_{2} X_{3} t^{3} h^{3} d a_{1} d a_{2} d a_{3}}{\left(h+\left(a_{1} X_{1}+a_{2} X_{2}+a_{3} X_{3}\right) t\right)^{4}} \\
& =\frac{h^{2}\left(h^{2}+\left(-4 X_{1}+3 X_{3}\right) h t+\left(3 X_{1}^{2}+3 X_{1} X_{2}-5 X_{1} X_{3}-X_{2} X_{3}+2 X_{3}^{2}\right) t^{2}\right)}{\left(h+\left(-3 X_{1}+X_{3}\right) t\right)\left(h+\left(-X_{1}-X_{2}+X_{3}\right) t\right)} .
\end{aligned}
$$

As promised, the denominator disappears (canceling the extra factor at numerator) after setting $X_{1}=h, X_{2}=2 h, X_{3}=3 h$; we get $\left(\right.$ as $\left.h^{2}=1\right)$

$$
\gamma_{\varphi}(t)=1+5 t+6 t^{2}
$$

i.e., $\gamma_{0}=1, \gamma_{1}=5, \gamma_{2}=6$. This says that $\varphi$ is generically 6 -to- 1 . Since the class of 
the monomials was $8 h$ to begin with, we see that the base locus of $\varphi$ contributes 58 to the intersection number $(8 h)^{2}$.

The integral appearing in Theorem 1.1 may be computed from a decomposition of the region $N_{\varphi}$ into simplices, including the positive coordinate directions as possible vertices (at infinity). We will use notation as in $[$ Alu15, §2.2]: in particular, if a simplex $T$ has $s+1$ finite vertices, its rank is $\operatorname{rk} T=s$; and the volume of $T$ is the normalized volume of the projection along its infinite directions. Also, we let $\operatorname{deg} T$ denote $h^{\operatorname{dim} V-\operatorname{rk} T} \cdot \prod X_{j}$, where the (intersection) product is taken over the complement of the infinite directions in $T$. We denote by $\underline{a}_{i}$ the vertex at infinity in the positive direction of the coordinate $a_{i}$.

Theorem 1.4. Let $\varphi$ be a r.c. monomial rational map. Then

$$
\gamma_{\varphi}(t)=\sum_{T} \widehat{\operatorname{Vol}}(T) \operatorname{deg}(T) t^{\mathrm{rk} T}
$$

where the sum ranges over the full-dimensional simplices $T$ in a triangulation of $N_{\varphi}$, with vertices chosen among vertices of $N_{\varphi}$ and infinite coordinate vertices.

Example 1.5. For the case illustrated in Example 1.3, a triangulation of $N_{\varphi}$ consists of the simplices $T_{1}=C \underline{a}_{1} \underline{a}_{2} \underline{a}_{3}, T_{2}=A C \underline{a}_{2} \underline{a}_{3}, T_{3}=A B \underline{a}_{1} \underline{a}_{3}, T_{4}=$ $A B C \underline{a}_{1}$. We have $\operatorname{rk} T_{1}=0 ; \operatorname{rk} T_{2}=\operatorname{rk} T_{3}=1 ; \operatorname{rk} T_{4}=2 ; \widehat{\operatorname{Vol}}\left(T_{1}\right)=1, \widehat{\operatorname{Vol}}\left(T_{2}\right)=3$, $\widehat{\mathrm{Vol}}\left(T_{3}\right)=\widehat{\mathrm{Vol}}\left(T_{4}\right)=1$ (as e.g., the projection of $T_{4}$ on the $a_{2} a_{3}$ plane is the triangle with vertices $(1,2),(0,2),(1,1))$; and $\operatorname{deg} T_{1}=h^{2-0}=1, \operatorname{deg} T_{2}=h^{2-1} \cdot X_{1}=1$, $\operatorname{deg} T_{3}=h^{2-1} \cdot X_{2}=2, \operatorname{deg} T_{2}=h^{2-2} \cdot X_{2} \cdot X_{3}=6$. According to Theorem 1.4,

$$
\gamma_{\varphi}(t)=1+3 \cdot 1 t+1 \cdot 2 t+1 \cdot 6 t^{2},
$$

agreeing with the previous computation.

Remark 1.6. The diagram determined by a choice of monomials and the volumes of the individual simplices of a decomposition are independent of the source variety $V$. Thus we could use the same data obtained in Example 1.5 to obtain the multidegree for the restriction $\varphi^{\prime}$ of the map defined in Example 1.3 to any degree- $d$ curve $V \subseteq \mathbb{P}^{2}$, as long as the restrictions of the curves $F_{1}=0$, $F_{2}=0, F_{3}=0$ to $V$ meet with regular crossings. Here, this means that $V$ should avoid the points of intersection of these curves. With such a choice of $V$ we have $\operatorname{deg} T_{1}=h^{1-0} \cdot V=d, \operatorname{deg} T_{2}=h^{1-1} \cdot X_{1} \cdot V=d, \operatorname{deg} T_{3}=h^{1-1} \cdot X_{2} \cdot V=2 d$, and $\operatorname{deg} T_{2}=h^{1-2} \cdot X_{2} \cdot X_{3} \cdot V=0$ (by dimension considerations). According to Theorem 1.4,

$$
\gamma_{\varphi^{\prime}}(t)=d+3 \cdot d t+1 \cdot 2 d t+1 \cdot 0 t^{2}=d+5 d t
$$

This says in particular that the degree of the image of a suitably general degree- $d$ curve $V \subseteq \mathbb{P}^{2}$ via $\varphi$ must be $5 d$. 
Remark 1.7. In the particular case where $V=\mathbb{P}^{r}$ and $X_{j}=$ coordinate hyperplanes, the multidegrees of rational monomial maps may be computed via toric methods by mixed volumes of Minkowski sums of polytopes (see e.g. [GSP06, §4] or [Dol, §3.5]). In particular, the top degree may be expressed as the ordinary (normalized) euclidean volume of a convex polytope; this is an instance of the Bernstein-Kouchnirenko theorem. The leading coefficient of (1.2) reproduces this result in this particular case, and extends it to the more general monomial maps on arbitrary projective varieties considered here, where toric techniques do not seem to be immediately applicable. Even for these very special monomial maps, it would be interesting to understand more fully the relation between the ordinary volumes appearing in Theorem 1.4 and the mixed volumes obtained by applying the Bernstein-Kouchnirenko theorem.

In the case $M=r=n-1, N_{\varphi}$ has one finite face which (if nondegenerate) is an $(n-1)$-simplex in $\mathbb{R}^{n}$. The map $\varphi$ is determined by the $n \times n$ matrix $\mathcal{M}_{\varphi}^{\prime}=\left(m_{i j}^{\prime}\right)$ whose rows $\underline{m}_{i}^{\prime}$ consist of the translated lattice points, as above. (So one row of $\mathcal{M}_{\varphi}^{\prime}$ is 0 .) We say that $\varphi$ is well-presented if the following requirement on $\mathcal{M}_{\varphi}^{\prime}$ is satisfied. Every choice of a set $I$ of indices $i_{1}, \ldots, i_{\ell}$ determines a projection $\mathbb{R}^{n} \rightarrow \mathbb{R}^{n-\ell}$ along the coordinate directions $a_{i_{1}}, \ldots, a_{i_{\ell}}$. We require that the projection of the Newton outer region of $\varphi$ be the Newton outer region determined by the projections of the rows $\underline{m}_{k}^{\prime}$ for $k \notin I$. (We also require a condition on the signs of certain minors of $\mathcal{M}_{\varphi}$; see $\S 4.2$.) For example, the standard Cremona transformation $\left(x_{1}: \cdots: x_{n}\right) \mapsto\left(\frac{1}{x_{1}}: \cdots: \frac{1}{x_{n}}\right)$ trivially satisfies this condition.

We prove that if $\varphi$ is well-presented, then the multidegree polynomial of $\varphi$ may be computed directly from the characteristic polynomial of the matrix $\mathcal{M}_{\varphi}^{\prime}$. The precise statement in the generality considered here is given in Theorem 4.11; for ordinary monomial rational maps $\varphi: \mathbb{P}^{n-1} \rightarrow \mathbb{P}^{n-1}$, the result may be stated as follows. Let

$$
\alpha:\left(x_{1}, \ldots, x_{n-1}\right) \mapsto\left(x_{1}^{a_{11}} \cdots x_{n-1}^{a_{1, n-1}}, \ldots, x_{1}^{a_{n-1,1}} \cdots x_{n-1}^{a_{n-1, n-1}}\right)
$$

be a morphism of tori, with $a_{i j} \in \mathbb{Z}$, inducing a rational map $\varphi: \mathbb{P}^{n-1} \rightarrow \mathbb{P}^{n-1}$. Let $A=\left(a_{i j}\right)$ be the matrix of exponents of $\alpha$, with characteristic polynomial $P_{A}(t)=\operatorname{det}(t I-A)$.

Theorem 1.8. If the monomial rational map $\varphi: \mathbb{P}^{n-1} \rightarrow \mathbb{P}^{n-1}$ is well-presented, then $\gamma_{\varphi}(t)=t^{n-1} P_{A}(1 / t)$.

It would be interesting to provide alternative characterizations of well-presented monomial rational maps. 


\section{$\S 2$. Proof of Theorem 1.1}

\section{$\S 2.1$. The multidegree class}

We work over an algebraically closed field.

Fulton-MacPherson intersection theory yields a direct relation between the multidegrees of a rational map $\varphi: \mathbb{P}^{r} \rightarrow-\mathbb{P}^{M}$ and the degrees of the Segre classes of the base scheme of $\varphi$ : see e.g. [Alu03, Proposition 3.1], [GSP06, Proposition 5], or [Dol, Proposition 2.3.1]. The case considered here requires the straightforward generalization of this relation to the case of rational maps $\varphi: V \rightarrow \mathbb{P}^{M}$, where $V$ is a subvariety of $\mathbb{P}^{r}$.

Notation: Let $V$ be a closed subvariety (or subscheme) of $\mathbb{P}^{r}, \mathscr{L}$ a line bundle on $V$, and let $\varphi: V \rightarrow \mathbb{P}^{M}$ be the rational map determined by a linear system in $H^{0}(V, \mathscr{L})$. Let $\Gamma \subseteq V \times \mathbb{P}^{M}$ be the closure of the graph of $\varphi$, and let $G$ be its 'shadow' in $V$ :

$$
G:=\pi_{*}\left(\left(1+H+H^{2}+\cdots\right) \cap[\Gamma]\right),
$$

where $H$ is the pull-back of the hyperplane class from the $\mathbb{P}^{M}$ factor, and $\pi: \Gamma \rightarrow V$ is the projection. This is the 'multidegree class' mentioned in the introduction; the multidegrees of $\varphi$ are the degrees of the components of $G$, viewed as classes in $\mathbb{P}^{r}$ :

$$
\gamma_{\varphi}(t)=\int\left(1+h t+h^{2} t^{2}+\cdots\right) \cap G=\sum_{\ell \geq 0}\left(h^{\operatorname{dim} V-\ell} \cdot G_{\ell}\right) t^{\ell},
$$

where $h$ is the pull-back of the hyperplane class from $\mathbb{P}^{r}$, and $G_{\ell}$ is the term of codimension $\ell$ in $G$. Thus, computing the multidegree polynomial is reduced to computing the multidegree class $G$.

Lemma 2.1. Let $i: S \subseteq V$ be the base scheme of the linear system defining $\varphi$. Then

$$
G=c\left(\mathscr{L}^{*}\right)^{-1}\left(\left([V]-i_{*} s(S, V)\right) \otimes_{V} \mathscr{L}^{*}\right)
$$

Here we are using the $\otimes$ notation introduced in $\S 2$ of [Alu94]: if $a$ is a class of codimension $p$ in $A_{*} V$, then $a \otimes_{V} \mathscr{L}^{*}$ denotes $c\left(\mathscr{L}^{*}\right)^{-p} \cap a$; the class $a \otimes_{V} \mathscr{L}^{*}$ is defined for all $a \in A_{*} V$ by extending this prescription by linearity. Propositions 1 and 2 in [Alu94] detail a few simple properties of this operation, freely used in what follows.

Proof of Lemma 2.1 (cf. [Alu03, Proposition 3.1]). If we identify $\Gamma$ with the blowup of $V$ along $S$, the class of the exceptional divisor $E$ is seen to equal $D-H$, where $D=\pi^{*} c_{1}(\mathscr{L})$. Thus 


$$
\begin{aligned}
G & =\pi_{*}\left(\frac{1}{1-H} \cap[\Gamma]\right)=c\left(\mathscr{L}^{*}\right)^{-1} \cap \pi_{*}\left(\frac{1-D}{1-H} \cap[\Gamma]\right) \\
& =c\left(\mathscr{L}^{*}\right)^{-1} \cap \pi_{*}\left(\left(\frac{1-D}{1-H} \cap[\Gamma]\right) \otimes_{\Gamma} \mathscr{L} \otimes_{\Gamma} \mathscr{L}^{*}\right) \\
& =c\left(\mathscr{L}^{*}\right)^{-1} \cap \pi_{*}\left(\left(\frac{1}{1+D-H} \cap[\Gamma]\right) \otimes_{\Gamma} \mathscr{L}^{*}\right) \\
& =c\left(\mathscr{L}^{*}\right)^{-1} \cap \pi_{*}\left(\left(\left(1-\frac{E}{1+E}\right) \cap[\Gamma]\right) \otimes_{\Gamma} \mathscr{L}^{*}\right) \\
& =c\left(\mathscr{L}^{*}\right)^{-1} \cap\left([V]-\pi_{*}\left(\frac{E}{1+E} \cap[\Gamma]\right) \otimes_{V} \mathscr{L}^{*}\right) \\
& =c\left(\mathscr{L}^{*}\right)^{-1}\left([V]-i_{*} s(S, V) \otimes_{V} \mathscr{L}^{*}\right)
\end{aligned}
$$

as stated.

\section{$\S 2.2$. R.c. monomial schemes and maps}

Now assume that $\varphi$ is monomial in the sense specified in $\S 1$ : the linear system defining $\varphi$ is generated by monomials $\mu_{0}, \ldots, \mu_{M}$ in sections $s_{j}$ of line bundles $\mathscr{L}_{j}, j=$ $1, \ldots, n$, on $V$; if $\mu_{i}=s_{1}^{m_{i 1}} \cdots s_{n}^{m_{i n}}$, we assume $\mathscr{L}_{1}^{\otimes m_{i 1}} \otimes \cdots \otimes \mathscr{L}_{n}^{\otimes m_{i n}} \cong \mathscr{L}$ for all $i$.

We let $X_{j}$ denote the zero-scheme of $s_{j}$. The zero-schemes of the monomials $\mu_{i}$ are effective divisors supported on the union of the $X_{j}$ 's, and the intersection of these divisors, i.e., the base scheme $S$ of $\varphi$, is a monomial scheme. The condition we require of the hypersurfaces $X_{j}$ is that monomial schemes defined with respect to $X_{1}, \ldots, X_{n}$ may be principalized by a sequence of blow-ups along codimension 2 monomial subschemes defined with respect to the proper transforms of the $X_{j}$ 's and the exceptional divisors in the blow-up sequence. C. Harris [Har15] proves that this condition holds if the hypersurfaces $X_{j}$ meet with regular crossings, i.e., for all $A \subseteq\{1, \ldots, n\}$ and all $p \in \bigcap_{i \in A} X_{i}$, the local equations for $X_{i}, i \in A$, form a regular sequence at $p$. Harris's theorem extends a result of R. Goward [Gow05, Theorem 2], which deals with the normal crossings case and under the assumption that $V$ is nonsingular. In the situation considered by Harris, neither $V$ nor the $X_{j}$ need to be smooth. For example, $V$ could be an arbitrarily singular subvariety of $\mathbb{P}^{r}$, and the $X_{j}$ could be obtained as intersections of $V$ with components of a divisor with simple normal crossings in $\mathbb{P}^{r}$, such that $V$ meets properly all strata of this divisor.

If the $X_{j}$ meet with regular crossings, then the base scheme $S$ is a r.c. monomial scheme in the sense of [Har15] and [Alu15], and we say that $\varphi$ is a r.c. monomial rational map. The theorems stated in the introduction hold under the assumption that $\varphi$ is r.c. monomial. 


\section{$\S 2.3$. Newton regions}

In the situation described in $\$ 2.2$, the base scheme $S$ of $\varphi$ is the subscheme of $V$ defined by the monomials $\mu_{0}, \ldots, \mu_{M}$. As in [Alu15], we associate with the monomials $\mu_{i}=s_{1}^{m_{i 1}} \cdots s_{n}^{m_{i n}}$ the lattice points $\left(m_{i 1}, \ldots, m_{i n}\right)$ in $\mathbb{R}^{n}$, and the 'Newton region' $N$ obtained as the (closure of the) complement in the positive orthant of the convex hull $N^{c}$ of the translations by $\mu_{i}$ of the positive orthants.

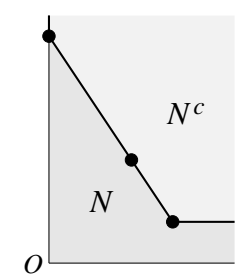

If $d_{j}=h^{\operatorname{dim} V-1} \cdot X_{j}$, then for every $i$ we have $\sum d_{j} m_{i j}=h^{\operatorname{dim} V-1} \cdot \sum m_{i j} X_{j}=$ $h^{\operatorname{dim} V-1} \cdot c_{1}(\mathscr{L}) \cap[V]=: d$. Therefore all the vertices $\left(m_{i j}\right)$ belong to the hyperplane with equation $d_{1} a_{1}+\cdots+d_{n} a_{n}=d$ in $\mathbb{R}^{n}$. We translate the vertices so that this hyperplane goes through the origin, for example by subtracting the coordinates of one vertex. (The choice of this pivoting monomial will be immaterial.)

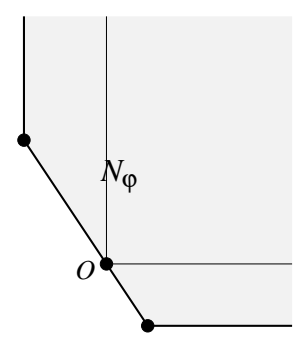

The 'Newton outer region' $N_{\varphi}$ is the corresponding translation of the region $N^{c}$.

Lemma 2.2. With notation as above,

$$
[V]-i_{*} s(S, V)=\int_{N^{c}} \frac{n ! X_{1} \cdots X_{n} d a_{1} \cdots d a_{n}}{\left(1+a_{1} X_{1}+\cdots+a_{n} X_{n}\right)^{n+1}}
$$

Proof. Since $S$ is r.c. monomial by assumption, by the main theorem in [Alu15] its Segre class in $V$ is evaluated by the integral over $N$ of the same rational function appearing on the right-hand side. Since the integral over the positive orthant is 1 (i.e., $[V])$, the integral over $N^{c}$ equals $[V]-i_{*} s(S, V)$ when viewed in $A_{*} V$, as stated. 


\section{$\S 2.4$. The main theorem}

By Lemmas 2.1 and 2.2, the multidegree class is given by

$$
G=c\left(\mathscr{L}^{*}\right)^{-1}\left(\int_{N^{c}} \frac{n ! X_{1} \cdots X_{n} d a_{1} \cdots d a_{n}}{\left(1+a_{1} X_{1}+\cdots+a_{n} X_{n}\right)^{n+1}} \otimes_{V} \mathscr{L}^{*}\right) .
$$

We can perform the $\otimes_{V}$ on the integrand. If $\mu=s_{1}^{m_{1}} \cdots s_{n}^{m_{n}}$ is the pivoting monomial, then $m_{1} X_{1}+\cdots+m_{n} X_{n}$ represents $c_{1}(\mathscr{L})$ (once the $X_{j}$ are replaced with the homonymous cycles). Using the simple properties of $\otimes_{V}$ (cf. [Alu94, §2]), we get

$$
\frac{n ! X_{1} \cdots X_{n}}{\left(1+a_{1} X_{1}+\cdots+a_{n} X_{n}\right)^{n+1}} \otimes_{V} \mathscr{L}^{*}=\frac{n ! c\left(\mathscr{L}^{*}\right) \cdot X_{1} \cdots X_{n}}{\left(1+\left(a_{1}-m_{1}\right) X_{1}+\cdots+\left(a_{n}-m_{n}\right) X_{n}\right)^{n+1}}
$$

and hence

$$
G=\int_{N^{c}} \frac{n ! X_{1} \cdots X_{n} d a_{1} \cdots d a_{n}}{\left(1+\left(a_{1}-m_{1}\right) X_{1}+\cdots+\left(a_{n}-m_{n}\right) X_{n}\right)^{n+1}} .
$$

Now, as $\left(a_{1}, \ldots, a_{n}\right)$ ranges over $N^{c}$, the translated point $\left(a_{1}-m_{1}, \ldots, a_{n}-m_{n}\right)$ ranges over $N_{\varphi}$. Therefore, we have

Theorem 2.3. With notation as above, the multidegree class of a r.c. monomial rational map $V \rightarrow \mathbb{P}^{n}$ is given by

$$
G=\int_{N_{\varphi}} \frac{n ! X_{1} \cdots X_{n} d a_{1} \cdots d a_{n}}{\left(1+a_{1} X_{1}+\cdots+a_{n} X_{n}\right)^{n+1}} .
$$

Theorem 2.3 is the primary result. To complete the proof of Theorem 1.1, it suffices to read off the degree $h^{\operatorname{dim} V-\ell} \cdot G_{\ell}$ from the components of the multidegree class, where $G_{\ell}$ has codimension $\ell$ in $V$. From (2.2), inserting a dummy variable $u$ to keep track of codimensions, we see that

$$
G_{0}+G_{1} u+G_{2} u^{2}+\cdots=\int_{N_{\varphi}} \frac{n ! X_{1} \cdots X_{n} u^{n} d a_{1} \cdots d a_{n}}{\left(1+\left(a_{1} X_{1}+\cdots+a_{n} X_{n}\right) u\right)^{n+1}} .
$$

Formally,

$$
\begin{aligned}
\gamma_{\varphi}(t) & =h^{\operatorname{dim} V} \cdot G_{0}+h^{\operatorname{dim} V-1} \cdot G_{1} t+h^{\operatorname{dim} V-2} \cdot G_{2} t^{2}+\cdots \\
& =h^{\operatorname{dim} V}\left(G_{0}+G_{1} \frac{t}{h}+G_{2} \frac{t^{2}}{h^{2}}+\ldots\right)
\end{aligned}
$$

Implementing this formal manipulation in (2.3) yields the integral given in (1.1), concluding the proof of Theorem 1.1. 


\section{§3. Proof of Theorem 1.4}

\section{$\S 3.1$. Generalized simplices}

Integrals such as the one appearing in Theorem 2.3 may be computed from a triangulation of the region $N_{\varphi}$. For us, a generalized simplex $T$ of rank $s$ in $\mathbb{R}^{n}$ is the subset spanned by $s+1$ affinely independent points $\underline{v}_{0}, \ldots, \underline{v}_{s}$, and $n-s$ positive coordinate directions $\underline{a}_{j_{1}}, \ldots, \underline{a}_{j_{n-s}}$ ('infinite vertices'). Thus,

$$
T=\left\{\sum_{i=0}^{s} \alpha_{i} \underline{v}_{i}+\sum_{k=1}^{n-s} \beta_{k} \underline{e}_{j_{k}} \mid \forall i, k: \alpha_{i} \geq 0, \beta_{k} \geq 0, \text { and } \sum_{i} \alpha_{i}=1\right\}
$$

where $\underline{e}_{1}=(1,0, \ldots, 0), \ldots, \underline{e}_{n}=(0, \ldots, 0,1)$. The (normalized) volume $\widehat{\operatorname{Vol}}(T)$ of a simplex is the normalized volume of the simplex obtained by projecting $T$ along its infinite directions. The simplex may degenerate when the vertices are affinely dependent; the volume of such an 'empty' simplex is 0 .

A simple calculus exercise yields the following result.

Lemma 3.1. If $T$ has finite vertices $\underline{v}_{i}=\left(v_{i 1}, \ldots, v_{i s}\right), i=0, \ldots, s$, and infinite vertices $\underline{a}_{j_{k}}, k=1, \ldots, n-s$, then

$$
\int_{T} \frac{n ! X_{1} \cdots X_{n} d a_{1} \cdots d a_{n}}{\left(1+a_{1} X_{1}+\cdots+a_{n} X_{n}\right)^{n+1}}=\frac{\widehat{\operatorname{Vol}}(T)}{\prod_{i=0}^{s}\left(1+v_{i 1} X_{1}+\cdots+v_{i n} X_{n}\right)} \cdot \frac{X_{1} \cdots X_{n}}{\prod_{k=1}^{n-s} X_{j_{k}}} .
$$

Proof. See [Alu15, Lemma 2.5].

\section{$\S 3.2$. Multidegree class and volumes of simplices}

The point now is that if $\left(v_{i 1}, \ldots, v_{i n}\right)$ is one of the translated monomials, i.e., one of the vertices of $N_{\varphi}$, then after evaluating the $X_{i}$ 's to the corresponding classes,

$$
v_{i 1} X_{1}+\cdots+v_{i n} X_{n}=0 .
$$

Indeed, $v_{i 1} X_{1}+\cdots+v_{i n} X_{n}$ is obtained by subtracting two classes both representing $c_{1}(\mathscr{L})$. Therefore, if $T$ is part of a triangulation of $N_{\varphi}$, and the finite vertices of $T$ are vertices of $N_{\varphi}$, then the contribution of $T$ to the multidegree class $G$ is simply (by Theorem 2.3)

$$
\widehat{\operatorname{Vol}}(T) \cdot \frac{X_{1} \cdots X_{n}}{\prod_{k=1}^{n-s} X_{j_{k}}}
$$

The factor

$$
X_{T}:=\frac{X_{1} \cdots X_{n}}{\prod_{k=1}^{n-s} X_{j_{k}}}
$$


for the generalized simplex $T$ is the product of the classes $X_{j}$ such that $\underline{a}_{j}$ is not an infinite vertex of $T$. As a class in $A_{*} V, X_{T}$ has codimension equal to the rank of $T$. Summarizing, we get

Corollary 3.2. With notation as above, the multidegree class for $\varphi$ is given by

$$
G=\sum_{T} \widehat{\operatorname{Vol}}(T) \cdot X_{T}
$$

where the sum is over the generalized simplices in a triangulation of $N_{\varphi}$, with finite vertices at vertices of $N_{\varphi}$.

Remark 3.3. The region $N_{\varphi}$ may be viewed as the convex hull of the (finite) translated monomials and of the (infinite) positive coordinate directions. As such, it always admits a triangulation whose simplices have vertices among these points (cf. [DLRS10, §2.2]).

\section{§3.3. Multidegree polynomial}

If an embedding of $V$ in a projective space $\mathbb{P}^{r}$ has been chosen, and $h$ is the restriction of the hyperplane class, it is now natural to let the degree of $T$ be the intersection number $h^{\operatorname{dim} V-\operatorname{rk} T} \cdot X_{T}$. By (3.1), we have

$$
\gamma_{\ell}=\sum_{\mathrm{rk} T=\ell} \widehat{\operatorname{Vol}}(T) \operatorname{deg}(T)
$$

where the sum is over (maximal dimension) simplices of fixed rank in a triangulation of $N_{\varphi}$. In other words,

$$
\gamma_{\varphi}(t)=\sum_{T} \widehat{\operatorname{Vol}}(T) \operatorname{deg}(T) t^{\mathrm{rk}(T)}
$$

concluding the proof of Theorem 1.4.

\section{$\S 4$. Well-presented rational maps}

\section{$\S 4.1$. The matrix of a rational map}

We now consider the $n \times(M+1)$-matrix $\mathcal{M}_{\varphi}$ whose rows are the vectors

$$
\underline{m}_{i}=\left(m_{i 1}, \ldots, m_{i n}\right)
$$

determined by the exponents of the monomials defining the rational map $\varphi$. We aim at identifying a condition under which the multidegree polynomial of $\varphi$ may be obtained directly from this matrix. We specialize to the case $M=n-1$, and renumber the monomials from 1 to $n$, so the matrix $\mathcal{M}_{\varphi}:=\left(m_{i j}\right)_{\substack{i=1, \ldots, n \\ j=1, \ldots, n}}$ is 
square. The motivating example is the case of dominant (ordinary) monomial maps $\mathbb{P}^{n-1} \rightarrow \mathbb{P}^{n-1}$, and monomial Cremona transformations in particular (cf. [Dol, $\S 3.5]$ ); we remind the reader that our context is more general, in that the source need not be projective space (or even nonsingular) and the monomials may be built on sections of line bundles (cf. $\S \S 1$ and 2).

\section{§4.2. Well-presented maps}

We say that $\varphi$ is well-presented by $\mathcal{M}=\mathcal{M}_{\varphi}$ if the following condition holds. For every subset $I=\left\{i_{1}, \ldots, i_{\ell}\right\} \subseteq\{1, \ldots, n\}$, we let $\mathcal{M}^{I}$ be the matrix obtained by removing the $i$-th row and column of $\mathcal{M}$ for all $i \in I$. We also let $\pi_{I}$ denote the projection $\mathbb{R}_{\geq 0}^{n} \rightarrow \mathbb{R}_{\geq 0}^{n-\ell}$ along the $i_{1}, \ldots, i_{\ell}$ directions. We require that

- The projection $\pi_{I}\left(N_{\varphi}\right)$ of the Newton outer region of $\varphi$ equals the Newton outer region determined by the projections $\pi_{I}\left(\underline{m}_{k}\right)$ for $k \notin I$.

- For all $I \subsetneq\{1, \ldots, n\}$, the determinant of $\mathcal{M}^{I}$ either is 0 or has sign $(-1)^{n-1-|I|}$.

Roughly, these conditions say that the ordered simplex determined by the rows of the matrix $\mathcal{M}$ is in sufficiently general position with respect to the coordinate directions.

Example 4.1. The standard Cremona transformation $\mathbb{P}^{2}-\rightarrow \mathbb{P}^{2},\left(x_{1}: x_{2}: x_{3}\right) \mapsto$ $\left(x_{2} x_{3}: x_{1} x_{3}: x_{1} x_{2}\right)$, with matrix

$$
\left(\begin{array}{lll}
0 & 1 & 1 \\
1 & 0 & 1 \\
1 & 1 & 0
\end{array}\right)
$$

is well-presented.

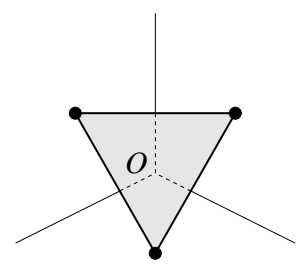

For example, for $I=\{3\}$, we see that the projection $(1,1)$ of the third row to the horizontal plane is in the region determined by the projections $(0,1)$ and $(1,0)$ of the other rows:

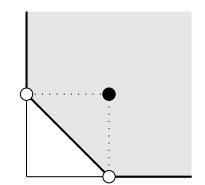


Also, $\operatorname{det} \mathcal{M}^{\{3\}}=\operatorname{det}\left(\begin{array}{ll}0 & 1 \\ 1 & 0\end{array}\right)=-1$, as required. The reader can verify that the conditions are satisfied for all choices of $I$.

On the other hand, the identity $\mathbb{P}^{2} \rightarrow \mathbb{P}^{2}$ is not well-presented.

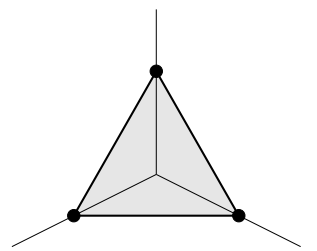

For example, for $I=\{3\}$ we have the following projection:

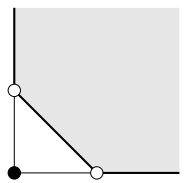

The projection of the third row is not in the outer region determined by the other two. Further, $\operatorname{det} \mathcal{M}^{\{3\}}=1 \neq(-1)^{1}$ in this case.

\section{§4.3. The Newton region of a well-presented rational map}

The main implication of the condition considered in $\S 4.2$ is the following description of the Newton region $N$ determined by a well-presented monomial map: this is the closure in the positive orthant of the complement of the outer region $N^{c}$ determined by the rows of $\mathcal{M}_{\varphi}$. (Recall that the region $N_{\varphi}$ is a translation of $N^{c}$.) For example, for the Cremona transformation in Example 4.1, the Newton region consists of three infinite parallelepipeds along the coordinate axes, and one tetrahedron connecting them:

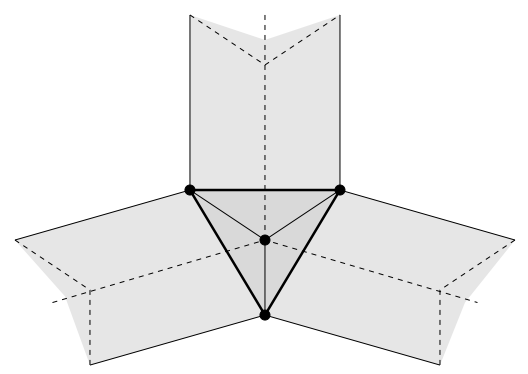

Lemma 4.2. If $\varphi$ is well-presented, then the corresponding Newton region is the (non-overlapping) union over all proper subsets $I=\left\{i_{1}, \ldots, i_{\ell}\right\} \subsetneq\{1, \ldots, n\}$ of the generalized simplices with infinite vertices at $\underline{a}_{i_{1}}, \ldots, \underline{a}_{i_{\ell}}$ and finite vertices at the origin and the rows $\underline{m}_{k}$ of $\mathcal{M}_{\varphi}$ for $k \notin I$. 
This statement is well illustrated by the the Cremona example depicted above. There are seven proper subsets of $\{1,2,3\}$; the tetrahedron in the middle corresponds to $I=\emptyset$; the three infinite simplices to the singletons; and the three simplices corresponding to the remaining three subsets are empty. More generally, the following picture (still for $n=3$ ) may help in visualizing the content of Lemma 4.2:

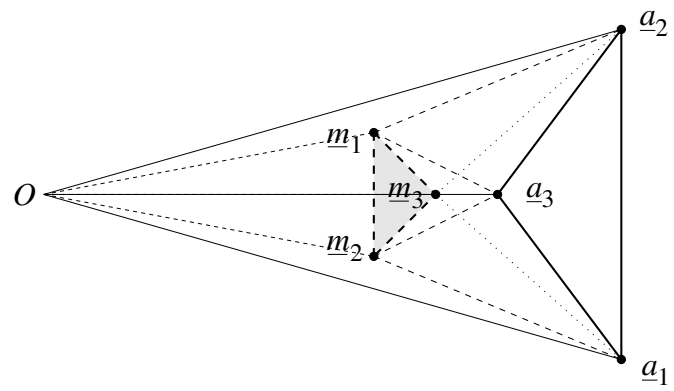

The positive orthant is represented by the simplex $O \underline{a}_{1} \underline{a}_{2} \underline{a}_{3}$, and $\underline{a}_{3}$ points towards the reader. The triangle $\underline{m}_{1} \underline{m}_{2} \underline{m}_{3}$ is contained in the orthant, and $\underline{m}_{3}$ points away from the reader. The region between the triangles $\underline{m}_{1} \underline{m}_{2} \underline{m}_{3}$ and $\underline{a}_{1} \underline{a}_{2} \underline{a}_{3}$ is the region $N^{c}$. The region $N$ is its complement, i.e., the union of the simplices $O \underline{m}_{1} \underline{a}_{2} \underline{a}_{3}, O \underline{m}_{1} \underline{m}_{2} \underline{a}_{3}$, etc., as prescribed by the lemma. In the Cremona case of Example 4.1 , the vertices $\underline{m}_{1}, \underline{m}_{2}, \underline{m}_{3}$ are on the faces $O \underline{a}_{2} \underline{a}_{3}, O \underline{a}_{1} \underline{a}_{3}, O \underline{a}_{1} \underline{a}_{2}$, respectively, so three of the simplices are degenerate.

Proof of Lemma 4.2. It is clear that $N$ may be decomposed as a union of simplices with vertices at the origin $O$, at some subset of infinite directions $\underline{a}_{i}, i \in I \subseteq$ $\{1, \ldots, n\}$, and at a subset of the rows of $\mathcal{M}$. Also, $I$ cannot consist of the whole $\{1, \ldots, n\}$, since the generalized simplex with vertices $O, \underline{a}_{1}, \ldots, \underline{a}_{n}$ is the whole positive orthant. For $I=\left\{i_{1}, \ldots, i_{\ell}\right\}$ properly contained in $\{1, \ldots, n\}$, we have to determine the set of $n-\ell$ rows $\underline{m}_{k}$ completing $O, \underline{a}_{i_{1}}, \ldots, \underline{a}_{i_{\ell}}$ to a simplex $T$ contained in $N$. For this, we project along $I$. For $i \in I, \pi_{I}\left(\underline{m}_{i}\right)$ is in the outer region determined by the projections $\pi_{I}\left(\underline{m}_{k}\right)$ for $k \notin I$, by the first requirement listed in $\S 4.2$. It follows that these latter $n-\ell$ rows must be the complementary set of vertices of $T$, and this is the assertion of the lemma.

\section{$\S 4.4$. The multidegree class of a well-presented map}

Lemma 4.2 and volume computations give the following result. Recall that $G$ denotes the multidegree class (cf. $\S 2.1$ ), and that for $I \subseteq\{1, \ldots, n\}, \mathcal{M}^{I}$ denotes the matrix obtained by removing the $i$-th row and column from $\mathcal{M}$ for all $i \in I$. 
Corollary 4.3. If $\varphi$ is r.c.-monomial and well-presented by the matrix $\mathcal{M}=\mathcal{M}_{\varphi}$, then

$$
G=c\left(\mathscr{L}^{*}\right)^{-1} \cap\left(\sum_{I \subseteq\{1, \ldots, n\}}(-1)^{n-|I|}\left(\operatorname{det} \mathcal{M}^{I}\right) \prod_{k \notin I} X_{k}\right) .
$$

Proof. By (2.1) in $\S 2.4$,

$$
G=\int_{N^{c}} \frac{n ! X_{1} \cdots X_{n} d a_{1} \cdots d a_{n}}{\left(1+\left(a_{1}-m_{n 1}\right) X_{1}+\cdots+\left(a_{n}-m_{n n}\right) X_{n}\right)^{n+1}},
$$

where we have used $\underline{m}_{n}$ as pivot. As $N$ is the complement of $N^{c}$ in the positive orthant, and the integral over the orthant is $c\left(\mathscr{L}^{*}\right)$, the decomposition of $N$ obtained in Lemma 4.2 and the formula for integrals over simplices (Lemma 3.1; remember that $O$ is a vertex of each simplex) give

$$
G=c\left(\mathscr{L}^{*}\right)^{-1} \cap\left(1-\sum_{I \subsetneq\{1, \ldots, n\}}\left(\widehat{\operatorname{Vol}} T_{I}\right) X_{T_{I}}\right),
$$

where $T_{I}$ is the simplex with vertices at the origin, $\underline{a}_{i}$ for $i \in I$, and $\underline{m}_{k}$ for $k \notin I$. Now $\widehat{\operatorname{Vol}}\left(T_{I}\right)$ is, by definition, the normalized volume of the simplex spanned by the projections of the finite vertices of $T_{I}$; thus, it equals \pm the determinant of the matrix obtained by replacing, for $i \in I$, the $i$-th row of $\mathcal{M}$ with the standard basis vector $\underline{e}_{i}$. In other words, this volume equals $\pm \operatorname{det} \mathcal{M}^{I}$, and by the second requirement listed in $\S 4.2$ we have

$$
\widehat{\mathrm{Vol}}\left(T_{I}\right)=(-1)^{n-1-|I|} \operatorname{det} \mathcal{M}^{I}
$$

for $I$ properly contained in $\{1, \ldots, n\}$. Thus

$$
G=c\left(\mathscr{L}^{*}\right)^{-1} \cap\left(1+\sum_{I \subsetneq\{1, \ldots, n\}}(-1)^{n-|I|}\left(\operatorname{det} \mathcal{M}^{I}\right) X_{T_{I}}\right) .
$$

The statement follows if we set $\operatorname{det} \mathcal{M}^{I}=1$ for $I=\{1, \ldots, n\}$ (the 'empty matrix').

Since the nonzero components of the class $G$ have codimension at most $n-1$, the right-hand side of (4.1) is necessarily a polynomial of degree less than $n$ in the $X_{j}$ 's, once these are evaluated to the corresponding classes in $A_{*} V$. As we will see, this fact has a more direct explanation.

We consider the matrix $\mathcal{M}_{\varphi}(X)$ whose rows are the vectors

$$
\left(m_{i 1} X_{1}, \ldots, m_{i n} X_{n}\right) \text {. }
$$

As usual, the $X_{j}$ 's are considered as parameters at first, and will eventually be evaluated to the corresponding classes in $A_{*} V$. For the considerations which follow, 
we impose the condition

$$
m_{11} X_{1}+m_{12} X_{2}+\cdots+m_{1 n} X_{n}=\cdots=m_{n 1} X_{1}+m_{n 2} X_{2}+\cdots+m_{n n} X_{n}
$$

Once the $X_{j}$ are replaced with the corresponding classes $c_{1}\left(\mathscr{L}_{j}\right)$ in $A_{*} V$, this common value is $c_{1}(\mathscr{L})$ by assumption. This amounts to the statement that the column vector $\left(\begin{array}{c}1 \\ \vdots \\ 1\end{array}\right)$ is an eigenvector of $\mathcal{M}_{\varphi}(X)$, with eigenvalue $c_{1}(\mathscr{L})$. Thus, the characteristic polynomial of $\mathcal{M}_{\varphi}(X)$ has a factor of $t-c_{1}(\mathscr{L})$ :

$$
\operatorname{det}\left(t I-\mathcal{M}_{\varphi}(X)\right)=\left(t-c_{1}(\mathscr{L})\right) \cdot Q(t)
$$

for a polynomial $Q(t)$ of degree $n-1$. On the other hand, evaluating the characteristic polynomial of $\mathcal{M}_{\varphi}(X)$ at $t=1$ gives the term in parentheses appearing in (4.1): indeed,

$$
\operatorname{det}\left(t I-\mathcal{M}_{\varphi}(X)\right)=\sum_{I \subseteq\{1, \ldots, n\}} t^{|I|}(-1)^{n-|I|}\left(\operatorname{det} \mathcal{M}^{I}\right) \prod_{k \notin I} X_{k}
$$

by elementary linear algebra. It follows that $G$ may be computed directly from the polynomial $Q(t)$ :

Corollary 4.4. If $\varphi$ is well-presented, and with notation as above, $G=Q(1)$.

Proof. By (4.4), this follows from (4.1) and (4.3): the factor $t-c_{1}(\mathscr{L})$ equals $c\left(\mathscr{L}^{*}\right)$ for $t=1$.

\section{$\S 4.5$. The multidegree class and a characteristic polynomial}

We will now identify $Q(t)$ itself as a characteristic polynomial. Let $\mathcal{M}_{\varphi}^{\prime \prime}(X)$ be the matrix obtained by subtracting the last row of $\mathcal{M}_{\varphi}(X)$ from the others, and discarding the last row and column:

$$
\left(\begin{array}{lll}
m_{11} X_{1} & m_{12} X_{2} & m_{13} X_{3} \\
m_{21} X_{1} & m_{22} X_{2} & m_{23} X_{3} \\
m_{31} X_{1} & m_{32} X_{2} & m_{33} X_{3}
\end{array}\right) \leadsto\left(\begin{array}{ll}
\left(m_{11}-m_{31}\right) X_{1} & \left(m_{12}-m_{42}\right) X_{2} \\
\left(m_{21}-m_{31}\right) X_{1} & \left(m_{22}-m_{42}\right) X_{2}
\end{array}\right)
$$

\section{Lemma 4.5.}

$$
Q(t)=\operatorname{det}\left(t I-\mathcal{M}_{\varphi}^{\prime \prime}(X)\right)
$$


Proof. This is also elementary linear algebra. If we perform a change of basis from the standard basis to $\underline{e}_{1}, \ldots, \underline{e}_{n-1}, \underline{e}_{1}+\cdots+\underline{e}_{n}$, then $\mathcal{M}_{\varphi}(X)$ is transformed into

$$
\left(\begin{array}{ccccc}
1 & 0 & \cdots & 0 & -1 \\
0 & 1 & \cdots & 0 & -1 \\
\vdots & \vdots & \ddots & \vdots & \vdots \\
0 & 0 & \cdots & 1 & -1 \\
0 & 0 & \cdots & 0 & 1
\end{array}\right) \cdot \mathcal{M}_{\varphi}(X) \cdot\left(\begin{array}{ccccc}
1 & 0 & \cdots & 0 & 1 \\
0 & 1 & \cdots & 0 & 1 \\
\vdots & \vdots & \ddots & \vdots & \vdots \\
0 & 0 & \cdots & 1 & 1 \\
0 & 0 & \cdots & 0 & 1
\end{array}\right)=\left(\begin{array}{cc}
\mathcal{M}_{\varphi}^{\prime \prime}(X) & 0 \\
* & c_{1}(\mathscr{L})
\end{array}\right)
$$

hence

$$
\operatorname{det}\left(t I-\mathcal{M}_{\varphi}(X)\right)=\left(t-c_{1}(\mathscr{L})\right) \cdot \operatorname{det}\left(t I-\mathcal{M}_{\varphi}^{\prime \prime}(X)\right) .
$$

The result follows by comparing with (4.3).

Corollary 4.6. With notation as above, and assuming that $\varphi$ is well-presented, the multidegree class of $\varphi$ is obtained by evaluating the characteristic polynomial for the matrix $\mathcal{M}_{\varphi}^{\prime \prime}(X)$ at $t=1$.

Remark 4.7. Note that $X_{n}$ does not appear in $\mathcal{M}_{\varphi}^{\prime \prime}(X)$, and hence in the expression for the multidegree class obtained in Corollary 4.6. This is not too surprising, given the redundancy built into the classes of the hypersurfaces $X_{i}$ (cf. (4.2)). Of course we could use the $i$-th row as pivot, and this would yield an expression for the multidegree class in which $X_{i}$ does not appear.

\section{§4.6. Monomial morphisms of tori}

The matrix $\mathcal{M}_{\varphi}^{\prime \prime}(X)$ has a compelling interpretation in the case of rational maps $\varphi: \mathbb{P}^{n-1} \rightarrow \mathbb{P}^{n-1}$ whose components are monomials in the homogeneous coordinates $x_{1}, \ldots, x_{n}$. Every such map may be obtained by homogenizing a monomial morphism of $(n-1)$-tori:

$$
\alpha:\left(x_{1}, \ldots, x_{n-1}\right) \mapsto\left(x_{1}^{a_{11}} \cdots x_{n-1}^{a_{1, n-1}}, \ldots, x_{1}^{a_{n-1,1}} \cdots x_{n-1}^{a_{n-1, n-1}}\right)
$$

with $a_{i j} \in \mathbb{Z}$. A homogenization may be performed (for example) by multiplying each monomial by a power of $x_{n}$ to obtain monomials of common degree 0 , then multiplying each monomial by a common factor to obtain nonnegative exponents throughout.

Example 4.8. Applying this procedure to the monomial map of tori

$$
\left(x_{1}, x_{2}, x_{3}\right) \mapsto\left(x_{1}^{-1} x_{3}, x_{2}^{-2}, x_{2} x_{3}\right)
$$


gives the following rational map $\mathbb{P}^{3} \rightarrow \mathbb{P}^{3}$ :

$$
\begin{aligned}
& \left(x_{1}: \cdots: x_{4}\right) \\
& \quad \mapsto\left(x_{1}^{-1} x_{3}: x_{2}^{-2} x_{4}^{2}: x_{2} x_{3} x_{4}^{-2}: 1\right)=\left(x_{2}^{2} x_{3} x_{4}^{2}: x_{1} x_{4}^{4}: x_{1} x_{2}^{3} x_{3}: x_{1} x_{2}^{2} x_{4}^{2}\right) .
\end{aligned}
$$

We say that $\alpha$ is well-presented if its homogenization is well-presented in the sense specified in $\S 4.2$. We are ready to prove Theorem 1.8 from the introduction:

Theorem 4.9. Let $\alpha$ be a map of $(n-1)$-tori, let $A=\left(a_{i j}\right)$ be the $(n-1) \times(n-1)$ matrix of exponents, let $P_{A}(t)=\operatorname{det}(t I-A)$ be the characteristic polynomial of $A$, and let $\gamma_{\alpha}(t)$ be the multidegree polynomial for the corresponding rational map $\mathbb{P}^{n-1} \rightarrow \mathbb{P}^{n-1}$. Assume that $\alpha$ is well-presented. Then

$$
\gamma_{\alpha}(t)=t^{n-1} P_{A}(1 / t)
$$

Proof. The matrix $h \cdot A$ obtained by multiplying each entry of $A$ by the hyperplane class $h$ equals the matrix $\mathcal{M}_{\varphi}^{\prime \prime}(X)$ for the rational map $\mathbb{P}^{n-1} \rightarrow \mathbb{P}^{n-1}$ obtained by homogenizing $\alpha$, and setting all $X_{j}$ to equal $h$. By Corollary 4.6 , the multidegree class equals $\operatorname{det}(I-h \cdot A)$, and the stated formula follows by formal manipulations.

Example 4.10. The standard Cremona transformation corresponding to the map of tori

$$
\left(x_{1}, \ldots, x_{n-1}\right) \mapsto\left(x_{1}^{-1}, \ldots, x_{n-1}^{-1}\right)
$$

is well-presented (Example 4.1). The exponent matrix is

$$
A=\left(\begin{array}{ccc}
-1 & \cdots & 0 \\
\vdots & \ddots & \vdots \\
0 & \cdots & -1
\end{array}\right)
$$

hence $P_{A}(t)=(t+1)^{n-1}$. According to Theorem 4.9, its multidegree polynomial is $\gamma_{\alpha}(t)=(1+t)^{n-1}$. Therefore its multidegrees are $\gamma_{\ell}=\left(\begin{array}{c}n-1 \\ \ell\end{array}\right)$ (cf. [GSP06, Theorem 2] and [Dol, §3.4]).

\section{§4.7. Multidegrees of well-presented r.c. monomial rational maps}

For more general well-presented r.c. monomial rational maps $V \rightarrow-\mathbb{P}^{n-1}$ based on $n$ hypersurfaces $X_{1}, \ldots, X_{n}$, consider (as in $\S \S 2$ and 3 ) the Newton outer region $N_{\varphi}$, whose vertices are the rows of the $n \times n$ matrix $\mathcal{M}_{\varphi}^{\prime}(X)$ of translations $\left(\underline{m}_{j}-\underline{m}_{n}\right) \cdot \underline{X}$ : 


$$
\begin{aligned}
&\left(\begin{array}{ccc}
m_{11} X_{1} & m_{12} X_{2} & m_{13} X_{3} \\
m_{21} X_{1} & m_{22} X_{2} & m_{23} X_{3} \\
m_{31} X_{1} & m_{32} X_{2} & m_{33} X_{3}
\end{array}\right) \\
& \sim\left(\begin{array}{ccc}
\left(m_{11}-m_{31}\right) X_{1} & \left(m_{12}-m_{32}\right) X_{2} & \left(m_{13}-m_{33}\right) X_{3} \\
\left(m_{21}-m_{31}\right) X_{1} & \left(m_{22}-m_{32}\right) X_{2} & \left(m_{23}-m_{33}\right) X_{3} \\
0 & 0 & 0
\end{array}\right)
\end{aligned}
$$

(Of course any row can serve as pivot.)

Theorem 4.11. Let $\varphi$ be well-presented, and let $\mathcal{M}_{\varphi}^{\prime}(X)$ be as above, with characteristic polynomial $P_{\mathcal{M}_{\varphi}^{\prime}(X)}(t)=\operatorname{det}\left(t I-\mathcal{M}_{\varphi}^{\prime}(X)\right)$. Then the multidegree polynomial of $\varphi$ is given by

$$
\gamma_{\varphi}(t)=h^{\operatorname{dim} V-n} t^{n} P_{\mathcal{M}_{\varphi}^{\prime}(X)}(h / t) .
$$

Identity (4.6) should be interpreted by computing the right-hand side formally; this yields an expression in $t$ whose coefficients are homogeneous polynomials of degree $\operatorname{dim} V$ in the variables $h, X_{1}, \ldots, X_{n}$. The statement is that evaluating the products as intersection products of the corresponding classes in $V$ gives the multidegree polynomial of $\varphi$.

Proof. The characteristic polynomial of $\mathcal{M}_{\varphi}^{\prime}(X)$ equals $t P_{\mathcal{M}_{\varphi}^{\prime \prime}(X)}(t)$, and it follows from Lemma 4.5 and Corollary 4.6 that

$$
P_{\mathcal{M}_{\varphi}^{\prime}(X)}(t)=t^{n} G_{0}+t^{n-1} G_{1}+\cdots+t G_{n-1},
$$

where $G_{\ell}$ is the term of codimension $\ell$ in $G$. Since

$$
\gamma_{\varphi}(t)=h^{\operatorname{dim} V} \cdot G_{0}+h^{\operatorname{dim} V-1} \cdot G_{1} t+\cdots
$$

the stated formula follows by formal manipulations of these expressions.

Example 4.12. Returning to the case in Example 1.3, $\varphi$ is well-presented, as the reader can easily verify. The matrices $\mathcal{M}_{\varphi}, \mathcal{M}_{\varphi}^{\prime}(X)$ are

$$
\left(\begin{array}{lll}
0 & 1 & 2 \\
2 & 0 & 2 \\
3 & 1 & 1
\end{array}\right), \quad\left(\begin{array}{ccc}
-3 X_{1} & 0 & X_{3} \\
-X_{1} & -X_{2} & X_{3} \\
0 & 0 & 0
\end{array}\right)
$$

We have $\operatorname{det}\left(t I-\mathcal{M}_{\varphi}^{\prime}(X)\right)=t\left(t+3 X_{1}\right)\left(t+X_{2}\right)$. According to Theorem 4.11,

$$
\gamma_{\varphi}(t)=h^{2-3} t^{3} \frac{h}{t}\left(\frac{h}{t}+3 X_{1}\right)\left(\frac{h}{t}+X_{2}\right)=h^{2}+\left(3 h \cdot X_{1}+h \cdot X_{2}\right) t+3 X_{1} X_{2} t^{2} .
$$


Since $X_{1}=h$ and $\operatorname{deg} X_{2}=2 h$ in this example, this recovers the result $\gamma_{\varphi}(t)=$ $1+5 t+6 t^{2}$ for the multidegree polynomial of $\varphi$, in agreement with the computations performed in Examples 1.3 and 1.5.

\section{Acknowledgements}

The author is grateful to Igor Dolgachev for useful conversations, particularly concerning the material in $\S 4$, and to the referee for a careful reading of the paper and for the suggestion to include Remark 1.6. The author's research is partially supported by a Simons collaboration grant.

\section{References}

[Alu94] P. Aluffi, MacPherson's and Fulton's Chern classes of hypersurfaces, Int. Math. Res. Notices 1994, no. 11, 455-465. Zbl 0839.14035 MR 1316973

[Alu03] Computing characteristic classes of projective schemes, J. Symbolic Comput. 35 (2003), 3-19. Zbl 1074.14502 MR 1956868

[Alu15] _ Segre classes as integrals over polytopes, J. Eur. Math. Soc., to appear; arXiv:1307.0830 (2015).

[DLRS10] J. A. De Loera, J. Rambau, and F. Santos, Triangulations, Algorithms Comput. Math. 25, Springer, Berlin (2010). Zbl 1207.52002 MR 2743368

[Dol] I. Dolgachev, Lectures on Cremona transformations. Ann Arbor and Rome 2010/11, http://www.math.lsa.umich.edu/ idolga/cremonalect.pdf.

[GSP06] G. Gonzalez-Sprinberg and I. Pan, On characteristic classes of determinantal Cremona transformations, Math. Ann. 335 (2006), 479-487. Zbl 1097.14011 MR 2221122

[Gow05] R. A. Goward, Jr., A simple algorithm for principalization of monomial ideals. Trans. Amer. Math. Soc. 357 (2005), 4805-4812. Zbl 1079.14021 MR 2165388

[GS] D. R. Grayson and M. E. Stillman, Macaulay2, a software system for research in algebraic geometry, http://www.math.uiuc.edu/Macaulay2/.

[Har15] C. Harris, Monomial principalization in the singular setting, J. Commutative Algebra, to appear; arXiv:1310.1261 (2015). 